\title{
OS EFEITOS DO USO CONCOMITANTE DE CAFEÍNA E CREATINA NOS EXERCÍCIOS FÍSICOS
}

\author{
THE EFFECTS OF CONCURRENT USE OF CAFFEINE AND CREATINE IN PHYSICAL EXERCISES
}

\author{
Raphael Silveira Nunes da Silva ${ }^{a^{*}}$, Adriana Marques Toigo ${ }^{b^{* *}}$ \\ araphaph7@gmail.com, badrytoigo@terra.com.br \\ *Academia Fitness Hall - Porto Alegre (RS), Brasil \\ **Centro Universitário La Salle - Canoas (RS), Brasil
}

Data de recebimento do artigo: 09/07/2015

Data de aceite do artigo: 24/09/2015

\section{RESUMO}

Introduçáo: a creatina e a cafeína encontram-se entre as substâncias mais utilizadas como recursos ergogênicos por aqueles que visam aumento da massa muscular, redução da gordura corporal e melhora no desempenho esportivo. Entretanto, pouco se sabe sobre os possíveis efeitos sinérgicos do consumo concomitante dessas substâncias. Objetivo: buscar evidências na literatura científica sobre os possíveis efeitos da administração dessas substâncias em sujeitos submetidos a exercícios físicos. Método: busca nas bases de dados LILACS, PubMed e SciELO, utilizando-se os termos "creatine", "caffeine", "resistance exercise" e "dietary supplementation". Resultados: onze estudos foram selecionados para análise dos resultados, pois enquadravam-se nos critérios de inclusão. Destes, apenas cinco tratavam do uso exclusivo de creatina associada a cafeína, e o restante, com outras substâncias comercializadas como suplementos alimentares. Os poucos estudos sobre o uso concomitante de creatina com cafeína se diferenciam bastante nos testes e protocolos de suplementação aplicados, tornando difícil evidenciar se o uso de tais substâncias pode realmente proporcionar efeitos benéficos aos seus consumidores. Conclusáo: os resultados de três artigos evidenciaram um efeito anulador da cafeína sobre os efeitos ergogênicos da creatina, ao passo que os demais apresentaram evidências contraditórias sobre as variáveis analisadas.

Palavras-chave: Cafeína; creatina; exercício.

\section{ABSTRACT}

Introduction: creatine and caffeine are among the most widely used substances as ergogenic resources by those looking to increase muscle mass, reduce body fat, and improve athletic performance. However, little is known about the possible synergistic effects of concomitant use of these substances. Objective: to search for evidence in the scientific literature about the possible effects of administration of these substances in subjects undergoing physical exercise. Method: a search in LILACS, SciELO, and PubMed databases, using the terms "creatine", "caffeine", "resistance exercise", and "dietary supplementation". Results: eleven articles were selected for analysis of the results, as they fulfilled the inclusion criteria. Of these, only five dealt with exclusive use of creatine associated with caffeine, and the remaining with other substances marketed as dietary supplements. The few studies on the concomitant use of creatine with caffeine differ greatly in the testing and supplementation protocols applied, making it difficult to conclude if the use of such substances can actually be beneficial to consumers. Conclusion: the results of three articles evidenced an annulment effect of caffeine on the ergogenic effects of creatine, and the others presented conflicting evidence on the variables analyzed.

Keywords: Caffeine; creatine; exercise. 


\section{Introdução}

Atualmente, diversas substâncias com potenciais efeitos ergogênicos são vendidas como suplementos alimentares. Tais suplementos sáo comercializados prometendo, principalmente, aumento da massa muscular, redução da gordura corporal e melhora no desempenho esportivo ${ }^{1,2}$. Entretanto, estudos relatam que o uso indiscriminado e mal informado dessas substâncias por adultos, adolescentes e até crianças pode gerar mais efeitos deletérios do que os benéficos esperados pelos seus consumidores. Assim, há uma emergente necessidade educacional em relação ao uso desses suplemento ${ }^{1-6}$.

Dentre as substâncias mais consumidas como recursos ergogênicos encontram-se a creatina e a cafeína ${ }^{7-9}$, e nenhuma dessas substâncias é proibida pela World Anti-doping Agency (WADA). Entretanto, o Comitê Olímpico Internacional (COI) considera doping uma concentração de cafeína maior que $12 \mu \mathrm{g} / \mathrm{mL}$ encontrada na urina, o equivalente ao consumo aproximado de $9 \mathrm{mg} / \mathrm{kg}$ de cafeína ou 5 a 6 xícaras regulares de café por um adulto de $70 \mathrm{~kg}$. Quanto à creatina, não há dosagem específica para ser considerada doping ${ }^{10,11}$.

\section{Cafeína}

A cafeína (Caf - 1,3,7-trimetilxantina) é uma substância lipossolúvel que pode ser completamente absorvida pelo intestino, atingindo um pico plasmático em 15 a 30 minutos, e transferida a praticamente todos os tecidos corporais. Após a circulação, sua biotransformaçáo ocorre em maior parte pelo citocromo P450 1A2, presente na matriz mitocondrial dos hepatócitos (cérebro e rins também apresentam tal enzima), sendo então degradada à paraxantina (1,7-dimetilxantina), teofilina (1,3-dimetilxantina) e teobromina (3,7-dimetilxantina), que são posteriormente excretadas na urina ${ }^{12-14}$.

Com ação psicotrópica, a cafeína pode ser encontrada nos grãos de café, chás, guaraná, sementes de cacau e bebidas contendo cola. Além dos produtos naturais, a cafeína também pode ser sintetizada e empregada em medicamentos, principalmente estimulantes, sedativos e redutores da fadiga ${ }^{10,13,15}$.

Aparentemente, atletas de alto rendimento que competem em esportes de longa duraçáo tendem a apresentar menores picos de concentraçáo de cafeína do que sujeitos fisicamente ativos (não atletas), assim como sujeitos com maior massa gorda apresentam concentraçóes de cafeína no sangue por períodos mais longos (aproximadamente 4 horas). Além disso, a ingestão regular de cafeína parece não influenciar as concentraçóes da substância no sangue. Portanto, parece que a identificação das condições ideais para garantir a disponibilidade de pico de cafeína no sangue e o entendimento de sua farmacocinética requer a consideração do estado de treinamento e composição corporal dos atletas ${ }^{16}$.

Na década de 1970, verificou-se que a cafeína promovia um melhor desempenho em exercícios aeróbicos de longa duraçáo devido ao aumento da taxa de oxidaçáo lipídica e pela economia e retardo do uso do glicogênio como fonte energética para a contração muscular ${ }^{17-19}$.

Mais recentemente, não foram constadas diferenças significativas nos efeitos da cafeína sobre a oxidação lipídica e o desempenho de indivíduos que foram submetidos a testes de carga progressiva em esteira ergométrica. Mesmo assim, verificou-se que os grupos que ingeriram bebida com cafeína permaneceram por mais tempo em atividade ${ }^{20}$.

Em exercícios intermitentes, de curta duração e alta intensidade, apesar dos poucos estudos existentes, algumas evidências descrevem a eficiência do consumo de cafeína em diminuir os efeitos da fadiga em protocolos de esportes específicos que exigem corridas repetidas e explosivas $^{21,22}$.

Foram propostos quatro possíveis mecanismos de açáo da cafeína que podem explicar seus potenciais efeitos ergogênicos em relação aos exercícios físicos: a) maior liberaçáo de cálcio do retículo sarcoplasmático; b) inibidor alostérico da enzima fosfodiesterase; c) antagonista dos receptores de adenosina $(A 1)$; e d) possível efeito sobre a atividade da bomba $\mathrm{Na}^{+}-\mathrm{K}^{+}$-ATPase, mantendo as concentraçôes de $\mathrm{K}^{+}$altas no meio intracelular e baixas no meio extracelular e, dessa forma, contribuindo para o retardo na fadiga ${ }^{14}$.

\section{Creatina}

A creatina ( $\mathrm{Cr}$ - ácido $\alpha$-metil guanidino acético) é um tripeptídeo capaz de se ligar a fosfatos inorgânicos (Pi) provenientes da hidrólise do ATP, por meio de uma reação reversível mediada pela enzima creatina quinase (CK), transformando-se assim em creatina-fosfato ( $\mathrm{CrP}$ - creatina fosforilada/fosfocreatina). Fisiologicamente, quando ocorre a contração muscular, a ATP é convertida em ADP e a CK catalisa a refosforilação da ADP em ATP a partir do fosfato contido na CrP. Isso torna a CrP a principal fonte energética fosfagênica para a ressíntese imediata de ATP durante exercícios de alta intensidade (exercícios anaeróbicos aláticos e de curta duração) e um importante tampão fisiológico do acúmulo de $\mathrm{H}^{+23}$.

De maneira exógena, a creatina pode ser obtida naturalmente através do consumo de carnes, principalmente peixes e gado ${ }^{24}$. Endogenamente, sua biossíntese ocorre a partir dos aminoácidos arginina, glicina e metionina em duas etapas: 1) nos rins, a arginina é convertida em ornitina, doando seu grupo guanidino (que 
contém nitrogênio) à glicina, que entáo se transformará em guanidinoacetato através de uma transaminação mediada pela enzima arginina:glicina-amidinotransferase (AGAT); 2) no fígado e pâncreas, a metionina tem seu radical metila extraído pela enzima $S$-adenosilmetionina, que entâo é ligado ao guanidinoacetato pela ação da enzima guanidinoacetato $\mathrm{N}$-metiltransferase (GAMT). Assim, a metilação do guanidinoacetato completa a síntese da molécula de creatina que, finalmente, será transportada pela corrente sanguínea aos tecidos alvos. A entrada da creatina nas células ocorre através de transportadores específicos, denominados "CreaT", dependentes da bomba $\mathrm{Na}^{+}-\mathrm{K}^{+25-27}$.

Como recurso ergogênico, a creatina constantemente é utilizada com o objetivo de aumentar a massa magra, força e velocidade e retardar a fadiga muscular quando acompanhada de treinamento resistido ${ }^{28-31}$.

Pesquisadores analisaram o desempenho e a composição corporal de ratos Wistar suplementados com creatina e submetidos a um treinamento de potência adaptado. Ao final do estudo, os grupos suplementados não demonstraram superioridade significativa em relação ao grupo placebo sobre o desempenho dos saltos. Por outro lado, a creatina, independente do treinamento de potência, foi capaz de aumentar a massa magra, assim como reduzir o percentual de gordura ${ }^{32}$.

Em um estudo conduzido anteriormente ${ }^{33}$, observou-se que sujeitos suplementados com creatina tiveram um aumento significativo no número de células satélites das fibras musculares e no número de mionúcleos, resultando em um aumento do volume muscular, principalmente na $4^{\mathrm{a}}$ e $8^{\mathrm{a}}$ semanas de treinamento de força. Além disso, no grupo suplementado com creatina, registrou-se um aumento no ganho de força máxima.

Ambas (creatina e cafeína) têm sido amplamente estudadas pela comunidade científica, isoladamente, concomitantes entre si ou até mesmo com outras substâncias, a fim de verificar possíveis efeitos ergogênicos sinérgicos ${ }^{10,25,28,34-40}$. Sendo assim, esta revisão propóe buscar evidências na literatura científica sobre os possíveis efeitos do consumo concomitante da creatina e da cafeína.

\section{Metodologia}

O presente estudo configurou-se como uma revisão sistemática da literatura, cujas bases de dados consultadas foram LILACS, PubMed e SciELO.

Nas referidas bases, as buscas foram realizadas utilizando-se os seguintes unitermos: "creatine", "caffeine", "resistance exercise", "dietary supplementation". O cruzamento dos termos supracitados, através do operador booleano "and", também foi executado. O período de pesquisa definido para a revisão foi limitado a 20 anos, até dezembro de 2014, e incluiu estudos publicados nas línguas portuguesa e inglesa.

Todos os cruzamentos efetuados estâo diagramados na Tabela 1:

Tabela 1: Termos e números de artigos localizados nas respectivas bases de dados.

\begin{tabular}{|c|c|c|}
\hline \multicolumn{3}{|c|}{1 - Creatine } \\
\hline \multicolumn{3}{|c|}{ 2-Caffeine } \\
\hline \multicolumn{3}{|c|}{ 3-Resistance Exercise } \\
\hline \multicolumn{3}{|c|}{ 4-Dietary Supplementation } \\
\hline LILACS & PUBMED & SCIELO \\
\hline $1-491$ & $1-24788$ & $1-298$ \\
\hline $2-301$ & $2-13902$ & $2-280$ \\
\hline $3-473$ & $3-14257$ & $3-273$ \\
\hline $4-688$ & $4-51009$ & $4-336$ \\
\hline 1 and $2-06$ & 1 and $2-138$ & 1 and $2-0$ \\
\hline 1 and $3-08$ & 1 and $3-360$ & 1 and $3-0$ \\
\hline 1 and $4-29$ & 1 and $4-942$ & 1 and $4-0$ \\
\hline 2 and $3-1$ & 2 and $3-59$ & 2 and $3-0$ \\
\hline 2 and $4-4$ & 2 and $4-316$ & 2 and $4-0$ \\
\hline 3 and $4-6$ & 3 and $4-446$ & 3 and $4-0$ \\
\hline 1 and 2 and $3-0$ & 1 and 2 and $3-10$ & 1 and 2 and $3-0$ \\
\hline 1 and 2 and $4-4$ & 1 and 2 and $4-45$ & 1 and 2 and $4-0$ \\
\hline 1 and 3 and $4-2$ & 1 and 3 and $4-116$ & 1 and 3 and $4-0$ \\
\hline 2 and 3 and $4-0$ & 2 and 3 and $4-11$ & 2 and 3 and $4-0$ \\
\hline $\begin{array}{l}1 \text { and } 2 \text { and } 3 \text { and } \\
4-0\end{array}$ & $\begin{array}{l}1 \text { and } 2 \text { and } 3 \text { and } \\
4-6\end{array}$ & $\begin{array}{c}1 \text { and } 2 \text { and } 3 \text { and } \\
4-0\end{array}$ \\
\hline
\end{tabular}

Como critérios de inclusão, foram considerados válidos artigos que tratassem diretamente de estudos experimentais em humanos e ratos. Ainda, os efeitos do suplemento de creatina deveriam ser testados concomitantemente com a administração de cafeína em pelo menos um dos grupos experimentais. Estudos em que os sujeitos testados ingeriram suplementos com outros compostos, mas que tivessem, fundamentalmente, a cafeína e a creatina em sua composição, também foram aceitos.

Não foram considerados artigos de revisão ou estudos de caso. 


\section{Resultados e Discussão}

Após a busca nos bancos de dados, um total de 11 trabalhos que se enquadraram nos critérios de inclusão foram encontrados. Destes, cinco tratavam de estudos exclusivos com creatina e cafeína e, os demais, do seu uso em conjunto com outras substâncias. Dos 11 estudos encontrados, apenas dois empregaram ratos em suas amostras.

O organograma (Figura 1) elucida os estudos selecionados.

Os resultados seguem a ordem cronológica de publicação e os detalhes das dosagens estáo resumidos na Tabela 2.

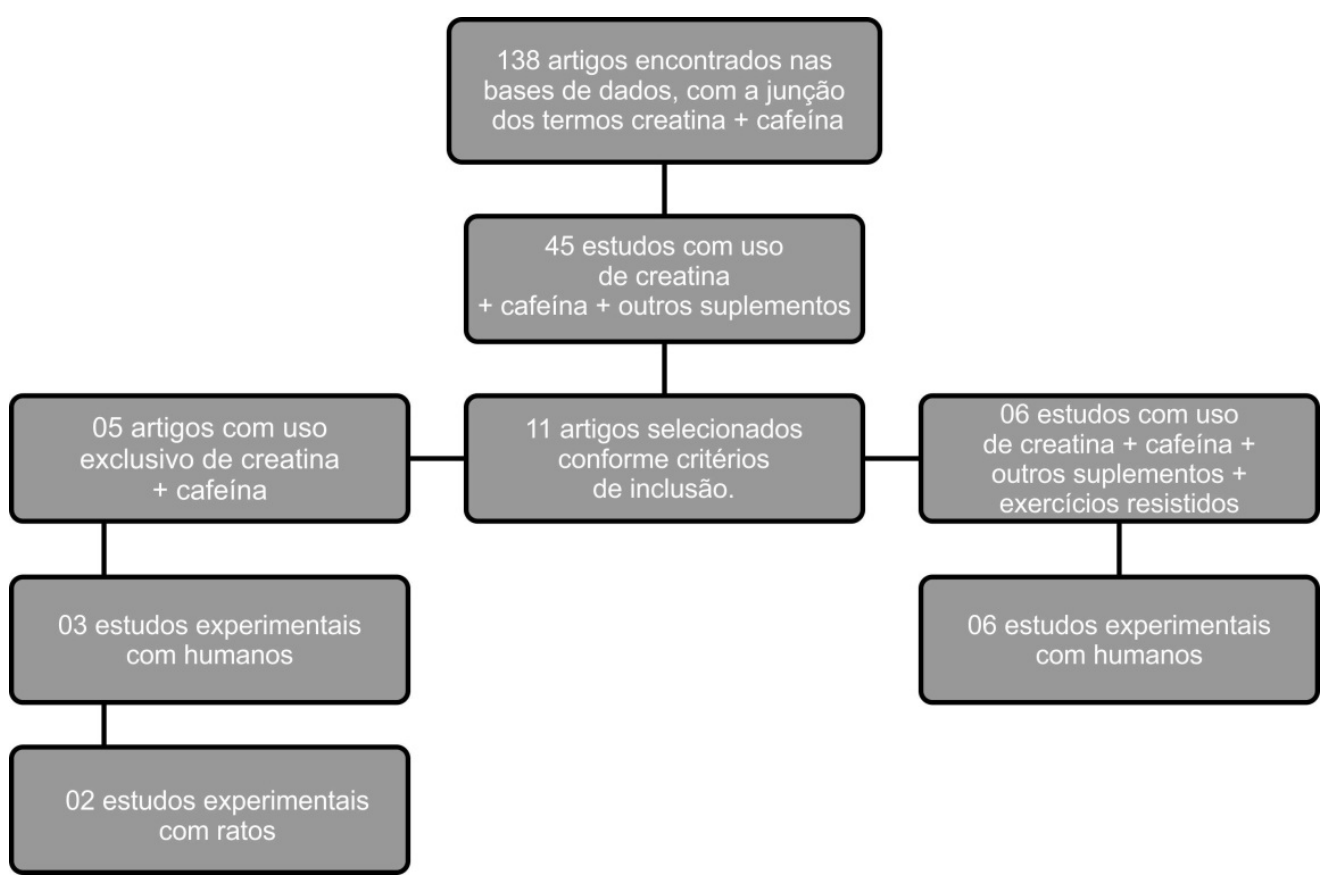

Figura 1: Organograma dos artigos incluídos.

Tabela 2: Resumo dos estudos analisados.

\begin{tabular}{|c|c|c|c|c|c|}
\hline \multirow[b]{2}{*}{$\begin{array}{l}\text { Autor(es) } \\
\text { (Ano) }\end{array}$} & \multirow[b]{2}{*}{$\begin{array}{l}\text { Desenho do } \\
\text { Estudo }\end{array}$} & \multicolumn{4}{|c|}{ Intervençáo } \\
\hline & & Amostra & $\begin{array}{l}\text { Protocolo de } \\
\text { Suplementaçáo }\end{array}$ & Variáveis & Resultados \\
\hline $\begin{array}{l}\text { Vandenberghe } \\
\text { et al. (1996) }\end{array}$ & $\begin{array}{l}\text { Randomizado } \\
\text { Controlado } \\
\text { Duplo-Cego } \\
\text { Crossover } \\
\text { Período }=08 \text { dias } \\
\text { Washout }=3 \text { sem }\end{array}$ & $\begin{array}{c}\text { Homens, } n=9 \\
20-23 \text { anos } \\
\text { Fisicamente Ativos } \\
\text { IMC = não relatado } \\
\text { Dieta padronizada }\end{array}$ & $\begin{array}{c}03 \text { grupos } \\
\mathrm{A}=\mathrm{Cr}-0,5 \mathrm{~g} \cdot \mathrm{kg}^{1} / 08 \mathrm{x} \text { ao } \\
\text { dia } / 06 \text { dias (dias } 02 \text { ao } 07) \\
\text { - última dose } 12 \mathrm{~h} \text { antes } \\
\text { dos testes } \\
\mathrm{B}=\mathrm{Caf}-0,5 \mathrm{~g} \cdot \mathrm{kg}^{1} \cdot \mathrm{dia} / 03 \\
\text { dias }(\text { dias } 05 \mathrm{ao} 07 \text { - última } \\
\text { dose } 20 \mathrm{~h} \text { antes dos testes } \\
\mathrm{C}=\mathrm{PL}-0.5 \text { glicose. } \mathrm{kg}^{1} . \\
\text { dia } / 06 \text { dias } \\
\text { *Refeiçóes padronizadas }\end{array}$ & $\begin{array}{l}\text { Pool de PCr muscular } \\
\text { (Espectropia }{ }^{31} \text {-P-NMR) } \\
\text { Torque máx. - dinâmico } \\
\text { e isométrico - extensão } \\
\text { unilateral de joelho (dina- } \\
\text { mômetro isocinético) }\end{array}$ & $\begin{array}{c}\text { Cr } \uparrow \text { torque máximo dinâmico } \\
\text { Caf anulou efeito ergogênico } \\
\text { da Cr na produçấo de força em } \\
\text { contraçôes dinâmicas }\end{array}$ \\
\hline $\begin{array}{l}\text { Hespel } \\
\text { et al. (2002) }\end{array}$ & $\begin{array}{l}\text { Randomizado } \\
\text { Controlado } \\
\text { Duplo-Cego } \\
\text { Crossover } \\
\text { Período }=08 \text { dias } \\
\text { Washout }=5 \mathrm{sem}\end{array}$ & $\begin{array}{c}\text { Homens, } n=9 \\
\text { Mulheres, } n=1 \\
21-24 \text { anos } \\
\text { Fisicamente ativos } \\
\text { IMC = não relatado }\end{array}$ & $\begin{array}{c}05 \text { grupos } \\
\begin{array}{c}\mathrm{A}=\mathrm{Cr}-5 \underset{\text { dias }}{5} \mathrm{~g} / 04 \mathrm{x} \text { ao dia } / 04 \\
\mathrm{~B}=\mathrm{Caf}-\underset{\mathrm{dias}}{0,5 \mathrm{~g} \cdot \mathrm{kg}^{1} \cdot \mathrm{dia} / 03} \\
\mathrm{C}=\mathrm{Cr}+\mathrm{Caf} \\
\mathrm{D}=\mathrm{CafA}-0.5 \mathrm{~g} \cdot \mathrm{kg}^{1}(1 \mathrm{~h} \\
\text { pré-teste }) \\
\mathrm{E}=\mathrm{PL}=\text { maltodextrina }\end{array}\end{array}$ & $\begin{array}{l}\text { Torque máx. - dinâmico } \\
\text { e isométrico - extensão } \\
\text { unilateral de joelho, esti- } \\
\text { mulado eletricamente (di- } \\
\text { namômetro isocinético) }\end{array}$ & $\begin{array}{c}\mathrm{Cr} \downarrow \text { tempo de descanso necessá- } \\
\text { rio para gerar força em contraçôes } \\
\text { musculares repetidas }\end{array}$ \\
\hline
\end{tabular}


Tabela 2: Continuação.

\begin{tabular}{|c|c|c|c|c|c|}
\hline $\begin{array}{l}\text { Autor(es) } \\
\text { (Ano) }\end{array}$ & $\begin{array}{l}\text { Desenho do } \\
\text { Estudo }\end{array}$ & Amostra & $\begin{array}{l}\text { Interve } \\
\text { Protocolo de } \\
\text { Suplementaçáo }\end{array}$ & Variáveis & Resultados \\
\hline $\begin{array}{l}\text { Doherty et.al. } \\
\text { (2002) }\end{array}$ & $\begin{array}{l}\text { Randomizado } \\
\text { Controlado } \\
\text { Duplo-Cego } \\
\text { Crossover } \\
\text { Período }=13 \text { dias }\end{array}$ & $\begin{array}{l}\text { Homens, } n=14 \\
\text { Idade média }=22,7 \\
\quad \text { anos } \\
\text { Fisicamente ativos } \\
\text { IMC médio }=22,78 \\
\text { VO } \text { máx médio }=^{2} \\
58.1^{1} \mathrm{~mL} \cdot \mathrm{kg}^{1} \cdot \mathrm{min}^{1}\end{array}$ & $\begin{array}{c}02 \text { grupos } \\
\mathrm{A}=\mathrm{Cr}\left(0,3 \mathrm{~g} \cdot \mathrm{kg}^{1} / 04 \mathrm{x} \text { ao }\right. \\
\text { dia/06 dias })+ \text { placebo (água } \\
\text { adoçada }-1 \text { h pré-teste }) \\
\mathrm{B}=\mathrm{Cr}\left(0,3 \mathrm{~g} \cdot \mathrm{kg}^{1} / 04 \mathrm{x} \text { ao }\right. \\
\text { dia/06 dias }+ \text { Caf }=0,5 \mathrm{~g} \cdot \mathrm{kg}^{1} \\
(1 \mathrm{~h} \text { pré-teste })\end{array}$ & $\begin{array}{c}\text { Escala de Borg } \\
\text { Amostra sanguínea } \\
\mathrm{VO}_{2} \text { máx (espirometria em } \\
\text { esteira ergométrica) }\end{array}$ & $\begin{array}{c}\text { Caf } \downarrow \text { taxa de percepção de esforço } \\
\text { Caf } \downarrow \text { efluxo de } \mathrm{K}^{+} \text {intracelular } \\
\text { Caf } \uparrow \text { tempo de corrida } \\
\text { Caf } \uparrow \mathrm{VO}_{2} \text { máximo }\end{array}$ \\
\hline $\begin{array}{l}\text { Fukuda et al. } \\
\text { (2010) }\end{array}$ & $\begin{array}{c}\text { Randomizado } \\
\text { Controlado } \\
\text { Duplo-Cego } \\
\text { Crossover } \\
\text { Período = } 9 \text { encon- } \\
\text { tros separados por } \\
24-48 \mathrm{~h}\end{array}$ & $\begin{array}{c}\text { Homens, } n=10 \\
\text { Idade média }=25,7 \\
\text { anos } \\
\text { Moderadamente } \\
\text { treinados } \\
\text { IMC médio }=23,98 \\
\text { VO máx médio }= \\
50,6 \text { mL.kg } \cdot \mathrm{min}^{1}\end{array}$ & $\begin{array}{c}02 \text { grupos } \\
\mathrm{A}=\mathrm{SUP}-\text { GameTime } \\
\text { min pré-exercício/teste) } \\
\mathrm{B}=\mathrm{PL}(30 \mathrm{~min} \\
\text { pré-exercício/teste) }\end{array}$ & $\begin{array}{l}\text { Corrida intermitente de } \\
\text { alta intensidade } \\
\mathrm{VO}_{2} \text { máx/VC/ } \\
\mathrm{CCA} \text { (espirometria em } \\
\text { esteira ergométrica) }\end{array}$ & $\begin{array}{c}\text { SUP } \uparrow \text { CCA } \\
\text { SUP } \uparrow \text { tempo até exaustáo }\end{array}$ \\
\hline $\begin{array}{l}\text { Smith et al. } \\
\quad(2010)\end{array}$ & $\begin{array}{l}\text { Randomizado } \\
\text { Controlado } \\
\text { Duplo-Cego } \\
\text { Período = } 6 \text { sem ( } 9 \\
\text { dias de treino) }\end{array}$ & $\begin{array}{c}n=24 \\
{ }^{*} \text { Homens } \\
\text { Idade média }=20,8 \\
\text { anos } \\
\text { IMC médio }=23,17 \\
\text { VO máx médio = } \\
3,71 \text { L.min } \\
\text { *Mulheres } \\
\text { Idade média }=21,5 \\
\text { anos } \\
\text { IMC = 21,52 } \\
\text { VO máx médio = } \\
\text { 2,57 L.min }\end{array}$ & 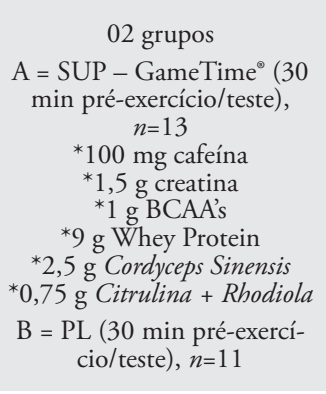 & $\begin{array}{l}\text { Corrida intermitente de } \\
\text { alta intensidade } \\
\mathrm{VO}_{2} \text { máx/VC/ } \\
\mathrm{CCA} \text { (espirometria em } \\
\text { esteira ergométrica) } \\
\text { Composiçáo corporal } \\
\text { (plestimografia com } \\
\text { deslocamento de ar) }\end{array}$ & $\begin{array}{c}\text { SUP } \uparrow \text { VC } \\
\text { SUP } \uparrow \text { volume de trabalho }\end{array}$ \\
\hline $\begin{array}{l}\text { Franco et al. } \\
\quad(2011)\end{array}$ & $\begin{array}{l}\text { Randomizado } \\
\text { Controlado } \\
\text { Período = } 06 \mathrm{sem}\end{array}$ & $\begin{array}{c}\text { Ratos Wistar } \\
\text { Idade }=07 \text { semanas } \\
\text { Peso médio } 142,7 \mathrm{~g} \\
\text { Dieta padronizada }\end{array}$ & $\begin{array}{c}08 \text { grupos }(n=10 / \mathrm{cada}) \\
\mathrm{A}=\text { Sedentário } \mathrm{PL} \\
\mathrm{B}=\text { Sedentário } \mathrm{Cr} \\
\mathrm{C}=\text { Sedentário Caf } \\
\mathrm{D}=\text { Sedentário Cr+Caf } \\
\mathrm{E}=\text { Exercício } \mathrm{PL} \\
\mathrm{F}=\text { Exercício } \mathrm{Cr} \\
\mathrm{G}=\text { Exercício Caf } \\
\mathrm{H}=\text { Exercício Cr+Caf } \\
\mathrm{Cr}=0,430 \mathrm{~g} \text { x } 07 \mathrm{dias}+ \\
0,143 \mathrm{~g} \cdot \mathrm{kg}^{1} \cdot{ }^{2} \text { dia x } 05 \mathrm{sem} \\
n=10 \\
\mathrm{Caf}=10 \mathrm{mg} \cdot \mathrm{kg}^{1} .05 \mathrm{sem} \\
\mathrm{PL}=20 \mathrm{~g} \text { de dieta }\end{array}$ & Composição Corporal & Caf $\downarrow \%$ de Gordura \\
\hline $\begin{array}{l}\text { Gonzalez et al. } \\
\text { (2011) }\end{array}$ & $\begin{array}{l}\text { Randomizado } \\
\text { Controlado } \\
\text { Duplo-Cego } \\
\text { Crossover } \\
\text { Experimentos = } \\
3 \text { dias } \\
\text { Washout }=72 \mathrm{~h}\end{array}$ & $\begin{array}{c}\text { Adultos Jovens } \\
\text { Homens } n=08 \\
\text { Com experiência em } \\
\text { treinamento resistido } \\
\text { Idade média }=20,6 \\
\text { anos } \\
\text { IMC médio }=25,2\end{array}$ & $\begin{array}{c}02 \text { grupos } \\
\text { SUP = Amino Impact } \\
\text { *2,05 g taurina, glucorono- } \\
\text { lactona e cafeína } \\
* 7,9 \mathrm{~g} \mathrm{BCAA} \text { 's } \\
\text { *5 g creatina } \\
2,5 \mathrm{~g} \beta \text {-Alanina } \\
\mathrm{PL}=500 \mathrm{~mL} \text { de água } \\
\text { adoçada com sucralose } \\
10 \text { min pré-exercícios/testes }\end{array}$ & $\begin{array}{l}\text { Produçáo de força e po- } \\
\text { tência (agachamento com } \\
\text { barra ou supino plano) } \\
\text { Questionário (percepçáo } \\
\text { de foco, energia e fadiga) }\end{array}$ & $\begin{array}{c}\text { SUP } \uparrow \text { número de repetiçōes } \\
\text { SUP } \uparrow \text { pico de potência } \\
\text { SUP = PL - sensaçōes de energia, } \\
\text { foco e fadiga }\end{array}$ \\
\hline $\begin{array}{l}\text { Franco et al. } \\
\quad(2012)\end{array}$ & $\begin{array}{l}\text { Randomizado } \\
\text { Controlado } \\
\text { Período = } 06 \mathrm{sem}\end{array}$ & $\begin{array}{c}\text { Ratos Wistar } \\
\text { Idade }=07 \text { semanas } \\
\text { Peso médio } 142,7 \mathrm{~g} \\
\text { Dieta padronizada }\end{array}$ & $\begin{array}{c}\mathrm{A}=\text { Sedentário } \mathrm{PL} \\
\mathrm{B}=\text { Sedentário } \mathrm{Cr} \\
\mathrm{C}=\text { Sedentário Caf } \\
\mathrm{D}=\text { Sedentário } \mathrm{Cr}+\mathrm{Caf} \\
\mathrm{E}=\text { Exercício } \mathrm{PL} \\
\mathrm{F}=\text { Exercício } \mathrm{Cr} \\
\mathrm{G}=\text { Exercício Caf } \\
\mathrm{H}=\text { Exercício Cr+Caf } \\
\mathrm{Cr}=0,430 \mathrm{~g} \times 07 \text { dias }+ \\
0,143 \mathrm{~g} \cdot \mathrm{kg}^{1} \cdot \mathrm{dia} \times 05 \mathrm{sem} \\
n=10 \\
\mathrm{Caf}=10 \mathrm{mg} \cdot \mathrm{kg}^{1} \cdot 05 \mathrm{sem} \\
\mathrm{PL}=20 \mathrm{~g} \text { de dieta }\end{array}$ & $\begin{array}{l}\text { Coleta de urina } \\
\text { Teste mecânico de } \\
\text { resistência de traçãó do } \\
\text { fêmur (N) }\end{array}$ & $\begin{array}{c}\text { Caf } \uparrow \text { concentraçáo de cálcio } \\
\text { urinário } \\
\text { Caf } \downarrow \text { força de fratura óssea } \\
\text { Cr+Caf = PL } \\
\text { Cr } \uparrow \text { força de fratura óssea }\end{array}$ \\
\hline
\end{tabular}


Tabela 2: Continuação.

\begin{tabular}{|c|c|c|c|c|c|}
\hline \multirow{2}{*}{$\begin{array}{l}\text { Autor(es) } \\
\text { (Ano) }\end{array}$} & \multirow{2}{*}{$\begin{array}{l}\text { Desenho do } \\
\text { Estudo }\end{array}$} & \multirow[b]{2}{*}{ Amostra } & \multicolumn{2}{|c|}{ Intervençáo } & \multirow[b]{2}{*}{ Resultados } \\
\hline & & & $\begin{array}{l}\text { Protocolo de } \\
\text { Suplementaçáo }\end{array}$ & Variáveis & \\
\hline $\begin{array}{l}\text { Ormsbee et al. } \\
\text { (2012) }\end{array}$ & $\begin{array}{l}\text { Randomizado } \\
\text { Controlado } \\
\text { Duplo-cego } \\
6 \text { sem de treina- } \\
\text { mento resistido } \\
-3 \mathrm{x} / \mathrm{sem}\end{array}$ & $\begin{array}{c}\text { Adultos jovens, } n=24 \\
\text { Fisicamente ativos } \\
\text { Idade média }=26,2 \\
\quad \text { anos } \\
\text { IMC médio }=25,83\end{array}$ & $\begin{array}{c}2 \text { grupos } \\
\text { SUP }= \\
\text { NO-Shotgun }(15 \text { min pré- } \\
\text {-treino) + NO-Synthesize } \\
\text { (pós-treino e diariamente) } \\
n=13 \\
\text { PL = Maltodextrina, } n=11 \\
\text { Experimento }=6 \text { semanas } \\
\text { Pós-teste }=36 \text { h após } \\
\text { suplementação }\end{array}$ & $\begin{array}{c}\text { Amostra sanguínea } \\
\text { (hormônios anabólicos) } \\
\text { Composiçáo Corporal } \\
\text { (DEXA) } \\
\text { Torque (potência) isomé- } \\
\text { trico e isocinético } \\
\text { Teste de Wingate } \\
\text { Teste de 1RM }\end{array}$ & $\begin{array}{c}\text { Grupo PL = Grupo SUP } \\
\text { (níveis hormonais/composição } \\
\text { corporal/desempenho muscular) } \\
\text { SUP } \uparrow \text { massa magra } \\
\text { SUP } \uparrow \text { pico de potência anaeróbi- } \\
\text { ca (Wingate) }\end{array}$ \\
\hline $\begin{array}{l}\text { Kedia et al. } \\
\text { (2014) }\end{array}$ & $\begin{array}{l}\text { Randomizado } \\
\text { Controlado } \\
\text { Duplo-Cego } \\
6 \text { sem de treina- } \\
\text { mento resistido } \\
-4 \mathrm{x} / \mathrm{sem}\end{array}$ & $\begin{array}{c}\text { Adultos jovens, } n=43 \\
\text { Fisicamente ativos } \\
\text { Idade média }=24,3 \\
\text { anos } \\
\text { IMC médio }=26,4\end{array}$ & $\begin{array}{c}2 \text { grupos } \\
\text { SUP }=\text { Craze }^{\mathrm{TM}} \\
(30 \text { min pré-treino ou dia- } \\
\text { riamente pela manhā }), n=22 \\
\mathrm{PL}=\text { Bebida isocalórica e } \\
\text { com cafeína, } n=21\end{array}$ & $\begin{array}{c}\text { Medida de pressáo arterial } \\
\text { e frequência cardíaca em } \\
\text { repouso } \\
\text { Biomarcadores sanguíneos } \\
\text { Teste de } 1 \text { RM } \\
\text { (supino) } \\
\text { Teste de potência e resis- } \\
\text { tência muscular } \\
\text { Composiçáo corporal } \\
\text { (DEXA) }\end{array}$ & $\begin{array}{c}\text { SUP } \uparrow \text { PAS (níveis seguros) } \\
\text { SUP } \downarrow \text { PAD (níveis seguros) } \\
\text { SUP } \uparrow \text { energia } \\
\text { SUP } \uparrow \text { concentração } \\
\text { PL = SUP nos biomarcadores } \\
\text { sanguíneos/composiçáo corporal/ } \\
\text { desempenho muscular }\end{array}$ \\
\hline
\end{tabular}

Abreviaçôes e símbolos: sem = semanas; $\mathrm{n}$ = número amostral; $\mathrm{IMC}=$ índice de massa corporal; $\mathrm{Cr}$ = creatina; Caf = cafeína; $\mathrm{PCr}=$ fosfocreatina; $\mathrm{BCAA}$ 's = aminoácidos de cadeia ramificada; $\mathrm{h}=$ horas; $\mathrm{SUP}=$ suplemento; $\mathrm{PL}=$ placebo; $\mathrm{PCr}=$ fosfocreatina; $\mathrm{NMR}=$ ressonância magnética nuclear; $\uparrow=$ aumentou; $\downarrow$ = diminuiu; $\mathrm{VO} 2$ máx = volume de oxigênio máximo; $\mathrm{VC}=$ velocidade crítica; $\mathrm{CCA}=$ capacidade de corrida anaeróbica; $\mathrm{kg}=$ quilogramas; $\mathrm{g}=$ gramas; $\mathrm{L}=$ litros; $\mathrm{mL}=$ mililitros, $\mathrm{N}=\mathrm{Newton}(\mathrm{s}) ; 1 \mathrm{RM}=$ uma execução máxima; $\mathrm{PAS}=$ pressão arterial sistólica; $\mathrm{PAD}$ = pressão arterial diastólica.

Apenas três estudos apresentaram evidências claras dos efeitos anuladores da cafeína sobre os efeitos benéficos da creatina ${ }^{41-43}$.

Vandenberghe et al. ${ }^{43}$ investigaram os possíveis efeitos sinérgicos do uso de creatina com cafeína em humanos. Os autores verificaram no grupo suplementado com creatina um aumento significativo de até $23 \%$ na produção de força máxima dinâmica, aplicada pelos músculos do quadríceps femural a um dinamômetro isocinético de extensão de joelho unilateral. Entretanto, surpreendentemente, os autores constataram que a cafeína anulou o efeito ergogênico da creatina, pois o grupo suplementado com ambas as substâncias demonstrou resultados muito semelhantes aos do grupo placebo.

Hespel et al. ${ }^{44}$ submeteram 10 adultos jovens a cinco protocolos distintos de suplementaçáo envolvendo o uso de creatina e/ou cafeína, a fim de verificar os efeitos da suplementação no desempenho exercido pelos músculos extensores de joelho estimulados por corrente elétrica. Os autores observaram que o tempo de contração e o torque máximo medidos em dinamômetro isocinético foram similares entre todas as condições de suplementação. Contudo, o grupo que ingeriu apenas creatina teve um tempo de descanso muscular significativamente menor (redução de 5\%) em relação aos demais grupos. Além disso, o grupo suplementado com cafeína de forma aguda (única dose 1 hora antes dos testes) também apresentou um menor tempo de descanso necessário para produzir contraçóes intermitentes, mesmo que esse efeito ainda tenha sido inferior ao do grupo suplementado exclusivamente com creatina. Dessa forma, os autores concluíram que houve um impacto negativo do consumo de cafeína no tempo de recuperaçáo muscular e que o efeito benéfico da suplementação de creatina foi anulado.

Franco et al. ${ }^{41}$ conduziram um estudo a fim de verificar os efeitos da suplementação de creatina e/ou cafeína no limiar de fratura óssea dos fêmures de ratos Wistar. O grupo exercitado que consumiu somente creatina apresentou maior resistência óssea à fratura (aproximadamente $120 \mathrm{~N}$ ) em relação aos demais grupos. O grupo exercitado que consumiu creatina e cafeína registrou força de fratura muito similar ao do grupo exercitado placebo (aproximadamente $110 \mathrm{~N}$ ). Os pesquisadores também verificaram que a suplementação com cafeína apresentou maiores concentrações de cálcio urinário (4,20 mg/24h) quando comparada aos animais que só consumiram placebo $(2,21 \mathrm{mg} / 24 \mathrm{~h})$ ou creatina $(3,3$ $\mathrm{mg} / 24 \mathrm{~h}$ ). Assim, o referido estudo corroborou os achados de outros autores em relação à possibilidade da creatina ter uma influência positiva na densidade e conteúdo mineral ósseo, mesmo que tais resultados tenham sido verificados somente em humanos idosos ${ }^{45}$.

Anteriormente, Franco et al. ${ }^{46}$ submeteram ratos machos jovens Wistar a um treinamento de saltos verticais adaptado (simulando um treinamento resistido 
intermitente) a fim de verificar as diferenças na composição corporal quando os mesmos consumiram dieta normal (placebo), creatina sozinha, cafeína sozinha, ou creatina e cafeína. Ao final das observaçóes, os pesquisadores verificaram que os animais suplementados com altas doses de creatina e cafeína e cafeína sozinha não apresentaram modificaçóes na composição corporal em relação aos grupos placebo. Contudo, a cafeína sozinha diminuiu a porcentagem de gordura (aproximadamente $1,5 \%$ e $1 \%$ ) quando comparada ao grupo suplementado com creatina sozinha ou creatina e cafeína, respectivamente.

Doherty et al..$^{34}$ submeteram 14 sujeitos a testes de corrida de alta intensidade e curta duração, com o objetivo de verificar os efeitos do consumo agudo de cafeína (única dose 1 hora pré-testes) após o uso de uma sobrecarga de creatina por 6 dias consecutivos. Como resultado, os pesquisadores verificaram que o consumo da cafeína foi capaz de, em relaçáo ao grupo placebo, aumentar significativamente o $\mathrm{VO}_{2 \text { máx }}\left(1,68\right.$ L.min $\left.{ }^{1}\right)$, aumentar o tempo de duração da corrida $(10 \%-23,8$ s), diminuir os níveis de percepção de esforço e, mesmo sem diferença significativa, diminuir o efluxo de $\mathrm{K}^{+}$intracelular. Os resultados desse estudo evidenciaram que a cafeína aumentou o $\mathrm{VO}_{2 \text { max }}$ e o tempo total de corrida, assim como diminuiu a percepçáo de esforço nos sujeitos suplementados com uma sobrecarga de creatina por 6 dias e que ingeriram uma dose aguda de cafeína 1 hora antes da realização dos testes.

Fukuda et al. ${ }^{47}$ examinaram os efeitos de um suplemento pré-treino contendo cafeína, creatina e aminoácidos na velocidade crítica (VC), na capacidade de corrida anaeróbia (CCA) e no tempo limite para atingir a exaustação em 10 sujeitos que realizaram corridas intermitentes e de alta intensidade em esteira. Os pesquisadores concluíram que os sujeitos suplementados apresentaram um aumento significativo de $10,8 \%$ na CCA e $12 \%$ no tempo limite para atingir a exaustão em relação ao grupo placebo. Entretanto, nenhuma diferença significativa foi observada na VC entre os grupos.

Smith et al..$^{39}$ examinaram os efeitos de um suplemento pré-treino (contendo cafeína, creatina e aminoácidos) combinado com 3 semanas de treinamento de corrida intervalada e de alta intensidade na composição corporal, no volume de treino e no desempenhos aeróbico e anaeróbico das corridas. Os pesquisadores verificaram que o grupo suplementado aumentou significativamente a VC $(2,9 \%)$ e o volume de treino $(11,6 \%)$, enquanto o grupo controle não apresentou melhoras significativas. Ainda, apesar de não ter apresentado diferenças significativas, aumentos positivos na massa corporal magra $(2,8 \%)$ e no $\mathrm{VO}_{2 \text { máx }}(10,3 \%)$ também foram relatados. Os autores concluíram que a combinação das respectivas substâncias consumida antes do treino, mesmo que em baixa dosagem, pode ser benéfica para aumentar o volume de treinamento e fazer a manutenção da massa magra.

Apesar dos estudos de Smith et al. ${ }^{39}$ e Fukuda et al. ${ }^{47}$ terem utilizado um composto de substâncias vendido como suplemento alimentar (GameTime ${ }^{\varpi}$ - CorrJensen Laboratories Inc., Aurora, Colorado, EUA), e mesmo que nesse composto a quantidade de cafeína e creatina seja inferior à administrada por Doherty et al. ${ }^{34}$, os três estudos empregaram como testes os exercícios de corridas de alta intensidade e curta duração, e os três estudos apontam para um aumento na capacidade total de corrida. Entretanto, os achados de Fukuda et al. ${ }^{47}$ se contrapuseram aos de Smith et al. ${ }^{39}$, pois os primeiros obtiveram benefícios na capacidade de corrida anaeróbia, enquanto que os outros autores demonstraram benefícios na velocidade crítica (VC), mesmo que os protocolos de testes tenham sido muito similares e os suplementos administrados nos dois estudos tenham sido idênticos. Dessa forma, os resultados são considerados inconclusivos sobre os reais efeitos benéficos da suplementação consumida.

Gonzalez et al. ${ }^{35}$ testaram os efeitos do consumo agudo de uma bebida energética contendo diversas substâncias (AminoImpact ${ }^{\mathrm{TM}}$ - Koach, Sport and Nutrition, Oviedo, FL, EUA), dentre elas creatina e cafeína, administrada 10 minutos pré-teste em oito homens jovens submetidos a exercícios resistidos multiarticulares (supino ou agachamento com barra) sobre o desempenho de produção de força, assim como nos níveis de energia, foco e fadiga. Os resultados indicaram um aumento significativo no número de repetiçôes (aproximadamente 3 repetiçóes) e na média do pico de potência (aproximadamente $40 \mathrm{~W}$ ) gerada durante os exercícios, comparado ao grupo controle. Os indivíduos não relataram diferenças nas sensaçóes de fadiga, energia e foco quando suplementados.

Spradley et al. ${ }^{40}$ submeteram indivíduos a diversos testes de tempo de reação, desempenho muscular, capacidades aeróbica e anaeróbica e percepção de fadiga, a fim de verificar os possíveis efeitos ergogênicos de uma mistura de substâncias, tais como cafeína, vitaminas do complexo B, aminoácidos, creatina e beta-alanina (Assault $^{\mathrm{TM}}$ - MusclePharm, Denver, Colorado, EUA), ingerida 10 minutos antes dos exercícios propostos. Como resultado, os autores constataram superioridade significativa em relação ao grupo placebo no desempenho de produção de força no exercício de leg press $(2$ repetiçóes), no tempo de reação (até 5\%) e nos níveis de energia, alerta e foco, apesar de nenhuma diferença ter sido constatada nos exercícios de corrida de alta intensidade. Tal diminuição da percepção de fadiga devido a um aumento dos níveis de energia corrobora os resultados apresentados no estudo de Kedia et $\mathrm{al}^{36}$, mas vão na direção oposta aos resultados de Gonzalez et al. ${ }^{35}$, 
que encontraram dados similares em ambos os grupos analisados.

Ormsbee et al. $^{38}$ propuseram investigar a eficácia de compostos pré e pós-treinos contendo mistura de substâncias ditas ergogênicas (NO-Shotgun ${ }^{\circ}$, contendo proteína extraída do soro do leite, caseína, aminoácidos de cadeia ramificada, creatina, beta-alanina e cafeína; e NO-Synthesize, contendo proteína extraída do soro do leite, caseína, aminoácidos de cadeia ramificada, creatina e beta-alanina - Vital Pharmaceuticals, Inc., Davie, FL, EUA) nos níveis de hormônios anabólicos, na composição corporal e no desempenho muscular de sujeitos que participaram de um protocolo de treinamento resistido periodizado durante 6 semanas. Verificou-se que os indivíduos do grupo controle apresentaram ganhos muito similares aos dos sujeitos suplementados em todas as variáveis analisadas (níveis de hormônios catabólicos, composição corporal, teste de força isométrica e isocinética, potência e força muscular). As únicas variáveis que se destacaram com diferenças estatisticamente significativas em relação ao grupo controle foram a composição corporal (aumento de massa magra em $4,7 \%$ em relação ao período pré-suplementação) e a produção de potência no teste de Wingate (aumento de $16,2 \%$ no pico de potência anaeróbica). Os autores concluíram que os suplementos pré e pós-treino estudados pareceram não apresentar vantagens ergogênicas em medidas de força muscular absoluta ou relativa e nos níveis hormonais, mas incitam ganhos na potência anaeróbica e no aumento da massa magra.

Kedia et al..$^{36}$ propuseram estudar os efeitos de um composto de substâncias ergogênicas (Craze ${ }^{\mathrm{TM}}$ - Driven Sports, NY, EUA) consumidas diariamente por meio da segurança de biomarcadores sanguíneos e da hemodinâmica aguda (pressão arterial sistêmica e frequência cardíaca) no desempenho muscular, na composição corporal e nas percepçóes de esforço em dois grupos submetidos a um protocolo periodizado de treinamento resistido por 6 semanas. Como resultado, os avaliadores constataram diferenças significativas entre os grupos apenas na percepção dos níveis de esforço e atenção (percepção de capacidade de trabalho). Assim, os autores concluíram que a suplementação estudada parece ser segura em relação aos níveis de pressão arterial e frequência cardíaca e nos biomarcadores sanguíneos, pois não apresentaram nenhuma alteração deletéria significativa nessas variáveis.

É importante ressaltar que a variedade de substâncias contidas nos suplementos administrados (a exemplo de "L-Arginina", "Citrulina Malato", "ß-Alanina", "Taurina", "Rhodiola Rosea", "Aminoácidos de Cadeia Ramificada" e diferentes formas de composiçáo e filtragem das proteínas do leite) junto com a cafeína e a creatina, principalmente nos estudos de Gonzalez et al. ${ }^{35}$, Kedia et al. ${ }^{36}$, Ormsbee et al ${ }^{38}$, Smith et al..$^{39}$, Spradley et al. ${ }^{40}$ e Fukuda et al. ${ }^{47}$, torna impossível identificar qual ou quais substâncias realmente podem estar proporcionando os possíveis efeitos ergogênicos observados nos respectivos estudos, do mesmo modo que dificultam a homogeneidade de testes aplicados, bem como a quantidade (dosagem) de substâncias existentes em cada um dos compostos. Outro ponto importante a destacar é que estudos mais atuais ${ }^{48}$ parecem esclarecer que protocolos antigos da suplementação de creatina, principalmente os que administram sobrecargas de dosagem (aproximadamente $20 \mathrm{~g} / \mathrm{dia}$ mais manutenção de $3-5 \mathrm{~g} / \mathrm{dia}$ por aproximadamente 28 dias $)^{31,44,49}$ não são mais eficientes do que doses de manutenção de aproximadamente $5 \mathrm{~g} /$ dia por períodos maiores. Essa variaçáo de protocolos talvez possa alterar a farmacocinética da cafeína em relação à creatina.

\section{Considerações finais}

Os poucos estudos sobre o uso concomitante de creatina com cafeína que se diferenciam bastante nos testes e protocolos de suplementação aplicados tornam difícil evidenciar se o uso de tais substâncias pode realmente proporcionar efeitos benéficos aos seus consumidores. Os resultados de três artigos realmente evidenciaram um efeito anulador da cafeína sobre os efeitos ergogênicos da creatina, principalmente em testes de desempenho muscular caracterizados por exercícios resistidos intermitentes. Os demais estudos apresentaram evidências contraditórias sobre as variáveis analisadas, principalmente quando a creatina e a cafeína foram administradas em conjunto com outras substâncias. Sugerimos, assim, que, em estudos futuros, protocolos de suplementação sejam mais atualizados e homogeneizados, caracterizados por análises de combinaçôes mais exclusivas em testes mais objetivos, para que os efeitos ergogênicos sinérgicos possam ser mais bem elucidados.

\section{Referências}

1. Alves C, Lima RVB. Dietary supplement use by adolescents. Jornal de Pediatria. 2009;85(4):287-94.

2. Goston JL, Correia MITD. Suplementos nutricionais: histórico, classificação, legislação e uso em ambiente esportivo. Nutrição em Pauta. 2009;98:1-7.

3. Hallak A, Fabrini S, Peluzio MCG. Avaliação do consumo de suplementos nutricionais em academias da zona sul de Belo Horizonte, MG, Brasil. Revista Brasileira de Nutrição Esportiva. 2007;1(2):55-60.

4. Linhares TC, Lima RM. Prevalência do uso de suplementos alimentares por praticantes de musculação nas 
academias de Campos dos Goytacazes/RJ, Brasil. Vértices. 2006;8(1):101-22.

5. Pereira RF, Lajolo FM, Hirschbruch MD. Consumo de suplementos por alunos de academias de ginástica em São Paulo. Revista de Nutrição. 2003;16(3):265-72.

6. Thein LA, Thein JM, Landry GL. Ergogenic aids. Physical Therapy. 1995;75(5):426-39.

7. Cook CJ, Crewther BT, Kilduff LP, Drawer S, Caviglio CM. Skill execution and sleep deprivation: effects of acute caffeine or creatine supplementation - a randomized placebo-controlled trial. J Int Soc Sports Nutr. 2011;8(2):1-8.

8. McDowall JA. Supplement use by young athletes. Journal of Sports Science \& Medicine. 2007;6(3):337-42.

9. Tarnopolsky MA. Caffeine and creatine use in sport. Annals of Nutrition and Metabolism. 2010;57(2):1-8.

10. American College of Sports and Medicine. Caffeine and exercise performance. Indianapolis, IN. [acesso em 06 jun. 2015]. Disponível em: http://www.acsm.org/docs/current-comments/caffeineandexercise.pdf

11. World Anti-doping Agency. The world anti-doping code: the 2014 prohibited list - International standard. [acesso em 23 mar. 2014]. Disponível em: http://www.wada-ama.org

12. Altimari LR, Cyrino ES, Zucas SM, Burini RC. Efeitos ergogênicos da cafeína sobre o desempenho físico. Rev. paul. Educ. Fís. 2000;14(2):141-58.

13. Altimari LR, Cyrilo ES, Zucas SM, Burini RC. Cafeína e performance em exercícios anaeróbios. Brazilian Journal of Pharmaceutical Sciences. 2006;42(1):186-90.

14. Braga LC, Alves MP. A cafeína como recurso ergogênico nos exercícios de endurance. Rev. Bras. Ciên. e Mov. 2000;8(3):33-7.

15. Guerra RO, Bernardo GC, Gutiérrez CV. Cafeína e esporte. Revista Brasileira de Medicina do Esporte. 2000;6(2):60-2.

16. Skinner TL, Jenkins DG, Leveritta MD, McGorma A, Bolam KA, Coombesa JS, et al. Factors influencing serum caffeine concentrations following caffeine ingestion. Journal of Science and Medicine in Sport. 2013;17(5):516-20.

17. Costill DL, Dalsky GP, Fink WJ. Effects of caffeine ingestion on metabolism and exercise performance. Medicine and science in sports. 1977;10(3):155-8.

18. Ivy JL, Costill DL, Fink WJ, Lower RW. Influence of caffeine and carbohydrate feedings on endurance performance. Medicine and Science in Sports. 1978;11(1):6-11.

19. Essig D, Costill DL, Van Handel PJ. Effects of caffeine ingestion on utilization of muscle glycogen and lipid during leg ergometer cycling. International Journal of Sports Medicine. 1980;1(2):86-90.

20. Brunetto D, Ribeiro JL, Fayh APT. Efeitos do consumo agudo de cafeína sobre parâmetros metabólicos e de desempenho em indivíduos do sexo masculino. Rev. bras. med. Esporte. 2010;16(3):171-5.

21. Del Coso J, Portillo J, Muñoz G, Abián-Vicén J, GonzalezMillán C, Muñoz-Guerra J. Caffeine-containing energy drink improves sprint performance during an international rugby sevens competition. Amino acids. 2013;44(6):1511-9.

22. Santos VGF, Bertuzzi R, Kiss MAPD, Lima-Silva AE. Efeito da suplementação de cafeína sobre o desempenho de exercícios intermitentes de alta intensidade. Acta brasileira do movimento humano - Revista de Educação Física. 2013;3(2):75-89.

23. Maughan R, Gleeson M, Greenhaff PL. Bioquímica do exercício e do treinamento. Barueri: Manole; 2000.

24. Peralta J, Amancio OMS. A creatina como suplemento ergogênico para atletas. Revista de Nutrição. 2002;15(1):83-93.

25. Persky AM, Brazeau GA. Clinical pharmacology of the dietary supplement creatine monohydrate. Pharmacological Reviews. 2001;53(2):161-176.

26. Koolman J, Röhm KH. Bioquímica: texto e atlas. Porto Alegre: Artmed; 2005.

27. Riegel ER. Bioquímica nutricional do exercício físico. São Leopoldo: Unisinos; 2005.

28. Nissen SL, Sharp RL. Effect of dietary supplements on lean mass and strength gains with resistance exercise: a meta-analysis. Journal of Applied Physiology. 2003;94(2):651-9.

29. Comitê Olímpico Internacional. Nutrition for Athletes. 2012. [acesso em 12 abril 2014]. Disponível em: http://www. olympic.org/documents/reports/en/en_report_833.pdf

30. Prestes J, Foschini D, Marchetti P, Charro M. Prescrição e periodização do treinamento de força em academias. São Paulo: Manole; 2010.

31. Vandenberghe K, Goris M, Van Hecke P, Van Leemputte $\mathrm{M}$, Vangerven L, Hespel P. Long-term creatine intake is beneficial to muscle performance during resistance training. Journal of Applied Physiology. 1997;83(6):2055-63.

32. Franco FSC, Natali AJ, Costa NMB, Lunz W, Gomes GJ, Carneiro Junior MA, et al. Efeitos da suplementação de creatina e do treinamento de potência sobre a performance e a massa corporal magra de ratos. Rev Bras Med Esporte. 2007;13(5):297-302.

33. Olsen S, Aagaard P, Kadi F, Tufekovic G, Verney J, Olesen $\mathrm{J}$, et al. Creatine supplementation augments the increase in satellite cell and myonuclei number in human skeletal muscle induced by strength training. The Journal of physiology. 2006;573(2):525-34.

34. Doherty M, Smith PM, Davison R, Hughes MG. Caffeine is ergogenic after supplementation of oral creatine monohydrate. Medicine and science in sports and exercise. 2002;34(11):1785-92.

35. Gonzalez AM, Walsh AL, Ratamess NA, Kang J, Hoffman JR. Effect of a pre-workout energy supplement on acute multi-joint resistance exercise. Journal of sports science \& medicine. 2011;10(2):261-6.

36. Kedia AW, Hofheins JE, Habowski SM, Ferrando AA, Gothard DM, Lopez HL. Effects of a pre-workout supplement on lean mass, muscular performance, subjective workout experience and biomarkers of safety. International journal of medical sciences. 2014;11(2):116-26. 
37. Lima FA, Sant'ana AEG, Ataíde TR, Omena CMB, Menezes MES, Vasconcelos SML. Café e saúde humana: um enfoque nas substâncias presentes na bebida relacionadas às doenças cardiovasculares. Revista de Nutrição. 2010;23(6):1063-73.

38. Ormsbee MJ, Mandler WK, Thomas WEG, Kimsey AW, Simonavice E, Panton LB, et al. The effects of six weeks of supplementation with multi-ingredient performance supplements and resistance training on anabolic hormones, body composition, strength, and power in resistance-trained men. J Int Soc Sports Nutr. 2012;9(1):49.

39. Smith AE, Fukuda DH, Kendall KL, Stout JR. The effects of a pre-workout supplement containing caffeine, creatine, and amino acids during three weeks of high-intensity exercise on aerobic and anaerobic performance. J Int Soc Sports Nutr. 2010;7(10):1-11.

40. Spradley BD, Crowley KR, Tai CY; Kendall KL, Fukuda $\mathrm{DH}$, Esposito EN, et al. Ingesting a pre-workout supplement containing caffeine, B-vitamins, amino acids, creatine, and beta-alanine before exercise delays fatigue while improving reaction time and muscular endurance. Nutr Metab (Lond). 2012;9:28-36.

41. Franco FSC, Costa Neusa MB, Oliveira TT, Gomes GJ, Silva KA, Natali AJ. Efeitos da suplementação com creatina e cafeína sobre a força de fratura óssea em ratos submetidos a exercício de saltos verticais. Revista da Educação Física/ UEM. 2012;23(1):105-14.

42. Hespel P, Op'T Eijnde B, Van Leemputte M. Opposite actions of caffeine and creatine on muscle relaxation time in humans. Journal of Applied Physiology. 2002;92(2):513-8.
43. Vandenberghe K, Gillis N, Van Leempute P, Van Hecke F, Vanstapel F, Hespel P. Caffeine counteracts the ergogenic action of muscle creatine loading. Journal of Applied Physiology. 1996;80(2):452-7.

44. Hespel P, Eijnde BO, Van Leempute M, Urso B, Greenhaff $\mathrm{PL}$, Labarque $\mathrm{V}$, et al. Oral creatine supplementation facilitates the rehabilitation of disuse atrophy and alters the expression of muscle myogenic factors in humans. The Journal of physiology. 2001;536(2):625-33.

45. Chilibeck PD, Chrusch MJ, Chad KE, Davison KS, Burke DG. Creatine monohydrate and resistance training increase bone mineral content and density in older men. The Journal of Nutrition, Health \& Aging. 2005;9(5):352-3.

46. Franco FSC, Costa NMB, Ferreira SA, Carneiro-Junior MA, Natali AJ. The effects of a high dosage of creatine and caffeine supplementation on the lean body mass composition of rats submitted to vertical jumping training. J Int Soc Sports Nutr. 2011;8(3):1-8.

47. Fukuda DH, Smith AE, Kendall KL, Stout JR. The possible combinatory effects of acute consumption of caffeine, creatine, and amino acids on the improvement of anaerobic running performance in humans. Nutrition research. 2010;30(9):607-14.

48. Antonio J, Ciccone V. The effects of pre versus post workout supplementation of creatine monohydrate on body composition and strength. Journal of the International Society of Sports Nutrition. 2013;10(1):36-43.

49. Hultman E, Söderlund K, Timmons JA, Cederblad G, Greenhaff PL. Muscle creatine loading in men. Journal of Applied Physiology. 1996;81(1):232-7.

\section{Como citar este artigo:}

Silva RSN, Toigo AM. Os efeitos do uso concomitante de cafeína e creatina nos exercícios físicos. Rev. Aten. Saúde. 2016;14(47): 89-98. 